\title{
Le recueil au Moyen Âge. La fin du Moyen Âge, sous la direction de Tania Van Hemelryck et de Stefania Marzano
}

\section{Antonella Amatuzzi}

\section{(2) OpenEdition}

\section{Journals}

\section{Edizione digitale}

URL: http://journals.openedition.org/studifrancesi/5884

DOI: $10.4000 /$ studifrancesi.5884

ISSN: 2421-5856

\section{Editore}

Rosenberg \& Sellier

\section{Edizione cartacea}

Data di pubblicazione: 1 mai 2011

Paginazione: 150-152

ISSN: 0039-2944

\section{Notizia bibliografica digitale}

Antonella Amatuzzi, «Le recueil au Moyen Âge. La fin du Moyen Âge, sous la direction de Tania Van Hemelryck et de Stefania Marzano», Studi Francesi [Online], 163 (LV | I) | 2011, online dal 30 novembre 2015, consultato il 13 janvier 2021. URL: http://journals.openedition.org/studifrancesi/5884 ; DOI: https://doi.org/10.4000/studifrancesi.5884

Questo documento è stato generato automaticamente il 13 janvier 2021.

\section{cc) (†)}

Studi Francesi è distribuita con Licenza Creative Commons Attribuzione - Non commerciale - Non opere derivate 4.0 Internazionale. 


\title{
Le recueil au Moyen Âge. La fin $\mathrm{du}$ Moyen Âge, sous la direction de Tania Van Hemelryck et de Stefania Marzano
}

\author{
Antonella Amatuzzi
}

\section{NOTIZIA}

Le recueil au Moyen Âge. La fin du Moyen Âge, sous la direction de Tania VAN HEMELRYCK et de STEFANIA MARZANo, Avec la collaboration d'Alexandra DIGNEF et de Marie-Madeleine DEPRoosT, Turnhout, Brepols («Texte, Codex \& Contexte», 9), 2010, 384 pp.

1 Questo volume riunisce i testi delle comunicazioni presentate al Troisième Colloque International du Groupe de Recherche sur le Moyen Français dell'Université catholique de Louvain (10-12 maggio 2007), che ruotano intorno alla questione delle raccolte manoscritte della fine del Medioevo, considerate in particolare nella loro dimensione materiale.

2 Hélène BASSo, Le poète à l'avenir effacé: Jean de Garencières, pp. 17-31, a partire dallo studio della collezione di componimenti lirici di Jean de Garencières (Paris, BnF, fr. 19139) propone una riflessione sulla poetica della raccolta. Se l'unione di frammenti disparati sembra trovare un'unità nell'autore, punto di riferimento essenziale, in realtà Garencières riunisce i suoi componimenti attorno ad un vuoto e la voce dell'autore si fa in disparte. Si tratta di un'opera aperta in cui è ogni lettore, nella sua individualità, ad essere sollecitato in prima persona.

Cynthia J. BROWN, La mise en œuvre et la mise en page des recueils traitant des femmes célèbres à la fin du Moyen Âge, pp.33-46, prende in esame quattro raccolte di testi ispirati direttamente o indirettamente da Anne de Bretagne che hanno come tema le donne celebri, per valutare il contributo della regina a queste antologie biografiche e la 
maniera in cui $\mathrm{i}$ testi vengono trasformati. Dapprima semplice dedicataria o committente, essa è man mano assimilata alle figure celebri dell'Antichità o della mitologia, diventando personaggio letterario. Ma questo omaggio alla donna serve in realtà ad innalzare l'immagine del marito. «La reine elle-même, d'une personne historique qui inspirait ou contrôlait la création de livres sur les femmes célèbres, est devenue une figure presque mythologique dont l'image était contrôlée par les poètes et artistes de cour» (p. 44).

Emma CAYLEY, Polyphonie et dialogisme: espaces ludiques dans le recueil manuscrit à la fin du Moyen Âge. La cas de trois recueils poétiques $d u X^{e}$ siècle, pp. 47-60, si occupa dei «débats» che si trovano in tre raccolte della tradizione manoscritta di Alain Chartier (Paris, BnF, fr. 1661; Paris, Arsenal, 3523; La Haye, Koninklijke Bibliotheek, 71 E 49) per tracciare dei legami tra l'opera di Chartier e i testi con i quali è trasmessa. Testo, paratesto e mise en recueil creano degli spazi concettuali; soprattutto il codice diventerebbe uno spazio ludico che mette in scena lo scambio tra il poeta e le sue comparse letterarie.

Paola CIFARELLI, Pierre Sala et le "Petit Livre d'Amour" (manuscrit Londres, BL, Stowe MS 955), pp.61-77, offre una lettura di questa «anthologie miniaturisée d'inspiration autobiographique» (p.63) che permette di ritrovarne una coerenza tematica e funzionale. Un'accurata interpretazione dei testi, che tiene conto dei giochi linguistici realizzati attraverso il dispositivo testo-immagini, permette di affermare infatti che non si tratta di materiali testuali eterogenei, che costituirebbero un libro d'amore, un omaggio cortese dell'amante alla dama amata, ma di una meditazione sulla vita di corte, una critica contro i vizi curiali.

Maria COLOMBO TIMELLI, Le ms. BAV Reg. Lat. 1716: un recueil de nouvelles? Quelques remarques sur le manuscrit des "Nouvelles" dites "de Sens", pp. 79-100, constata la mancanza di studi d'insieme sul ms. BAV Reg. Lat. 1716 (considerato generalmente una collezione di nouvelles, secondo il titolo che precede il primo di questi testi). Ipotizza quindi che ciò sia dovuto alla difficoltà che esiste per noi nel classificare come "novelle" questi scritti, che sono in realtà, come dimostra un attento esame del codice, estremamente vari ed eterogenei. Bisogna quindi supporre che si tratti di una raccolta in cui ai primi testi, che possono effettivamente rientrare nel genere novellistico, se ne accompagnano altri che appartengono ad altre tipologie di narratio brevis.

Olivier DELSAUX, (D)ebat pour recueil en noir majeur. La supériorité du ms.-recueil sur le ms. d'auteur pour l'approche d'un texte poétique en moyen français, pp. 101-111, studia il Debat de la noire et de la tannée, scritto verso il 1457 alla corte di Blois, tramandato in un manoscritto monotestuale (Paris, BnF, fr. 25420 [A]) e in tre raccolte (il Jardin de Plaisance, il ms. Paris, BnF, Rotschild 2798 e il ms. Chantilly, Bibliothèque du Château, 685) per arrivare alla conclusione che il testo acquista una dimensione molto diversa quando lo si mette in relazione di contiguità con altri. In particolare, la presenza di opere di Alain Chartier tende a smorzare la serietà delle lamentele d'amore e trasformerebbe il Debat in una sorta di gioco di società; la vicinanza con opere teatrali consente di immaginare che il Debat sia stato messo in scena. Il manoscritto-raccolta fornisce inoltre elementi utili per ipotizzare l'attribuzione dell'opera.

8 Estelle DOUDET, L'identité bourguignonne au temps des Habsbourg. Mise en recueil et littérature de circons-tance dans le manuscrit de Manchester, J. Rylands University Library, French 144, pp.113-123, mostra come il ms. in questione, che contiene ventuno opere poetiche e una cronaca versificata riguardante la storia della Borgogna negli anni 1467-1553, rappresenti il mezzo attraverso il quale il suo proprietario (Jean d'Haffrengues, 
fondatore di una celebre dinastia di mercanti) intende integrarsi nel luogo in cui vive, scegliendo delle opere che dichiarano la sua lealtà verso il potere borgognone, nonché il suo interesse per la storiografia della corte.

Barbara FERRARI, La "Légende dorée" du ms. Paris, BnF, fr. 23114, traduction anonyme pour Béatrice de Bourgogne, pp.125-135, dopo aver rammentato la tradizione manoscritta delle versioni francesi della Legenda aurea, si sofferma sul légendier trasmesso dal ms. Paris, BnF, fr. 23114, eseguito per Beatrice di Borgogna. Esso non è una trasposizione fedele in francese dell'originale latino ma una rielaborazione del modello, con alcune inserzioni che permettono di collocare l'origine della traduzione nella Francia centrooccidentale e alcuni indizi linguistici e codicologici che attesterebbero il "viaggio" del manoscritto verso il Nord.

Marie JENNEQUIN, Une filiation littéraire inscrite dans le manuscrit? Le "Livret sommaire" de Jean -Lemaire de Belges, pp. 137-153, a proposito del manoscritto Paris, BnF, nouv. acq. fr. 4061 (risalente al 1498 e realizzato da «maistre Regnault» su ordine di Jean Lemaire de Belges), sostiene che la scelta dei testi e la loro disposizione nella raccolta attestano un progetto intellettuale preciso da parte di Lemaire. Egli intende rivendicare una continuità, nell'ambito della storiografia borgognona, rispetto ai suoi due maestri Molinet e Chastelain (che vuole eguagliare e superare) e propone un itinerario spirituale teso ad una sintesi, una concordia tra la lingua latina e la francese, tra la cultura latina e la cultura medievale.

11 Nelly LABÈRE, "En la fourme et la maniere» des "Cent nouvelles nouvelles" de Philippe de Vigneulles, pp.157-177, riflette sull'organizzazione della materia nelle Cent nouvelles nouvelles. Nella raccolta, troppo spesso considerata un insieme eterogeneo, l'A. coglie un'unitarietà e una ricerca di organizzazione secondo una logica tematica e numerica. Nello spazio della novella, attualizzando la materia tradizionale, Vigneulles moltiplica il numero di storie, dando senso e fecondità al gesto della compilazione.

12 Sylvie LEFÈVRE, Un recueil du Xve siècle: le Mignon, pp. 179-198, riferendosi al manoscritto Paris, BnF, fr. 9186, designato in un ex-libris autografo come mignon (per indicare il libro preferito), nota come il nome scelto per la raccolta rinvii esplicitamente alla tecnica di assemblaggio usata. Il confronto con altri mignons apparentati prova inoltre come queste raccolte abbiano una configurazione estremamente variabile e fugace: il rapporto privilegiato che il possessore ha col manoscritto ne condiziona la fattura, personalizzandolo.

13 Margarida MADUREIRA, Le Recueil d'auteur au XIV siècle: Guillaume de Machaut et la compilation de ses œuvres, pp. 199-211, indaga sul manoscritto Paris, BnF, fr. 1586 (C), la più antica raccolta di opere di Machaut giunta fino a noi, in cerca dei segni della presenza della nozione d'autore. La consapevolezza che il poeta acquisisce della sua funzione e del valore delle sue opere evolve in relazione con il suo ruolo nella società. Così Machaut può permettersi di centralizzare la produzione di copie delle sue opere essenzialmente dopo aver preso possesso del canonicato di Reims, poiché gode allora di una certa libertà e indipendenza di mezzi.

14 Virginie MINET-MAHY, Polyphonie et problèmes de langage dans l'album poétique de Charles d'Orléans (Paris, BnF, fr. 25458), pp. 213-232, sulla base di osservazioni testuali puntuali, evidenzia le particolarità polifoniche (cambiamenti di punti di vista, effetti di eco...) del codice, che testimonia un mutamento nel lirismo di Charles d'Orléans. Dalla narrazione dell'io si passa al dinamismo del «débat», dello scambio verbale, e si assiste alla 
dissoluzione dell'io del poeta dietro la voce dell'altro, tendenza che caratterizzerà il Rinascimento segnando l'evoluzione della poesia verso la modernità.

Gilbert ouY, Deux frères à l'cuvre: Charles d'Orléans et Jean d'Angoulême compositeurs de recueils, pp.233-251, concentra l'attenzione sulle raccolte che i due fratelli hanno composto o hanno fatto copiare durante la loro prigionia in Inghilterra e da cui si possono trarre importanti informazioni. Innanzi tutto si ha la prova che Charles e Jean vissero assieme a Londra, dove frequentavano la biblioteca dei Greyfriars; molti manoscritti appaiono in effetti intrisi della spiritualità francescana inglese. Per il tramite dei francescani inoltre i due prigionieri vennero in contatto con le opere di Gerson.

Tiziano PACCHIARotTI, Les manuscrits du "Matheolus" et leur réception, pp. 253-261, indagando la tradizione manoscritta delle Lamentations, mostra come la percezione del testo abbia subito una trasformazione: si supera la logica anti-matrimoniale per giungere ad un discorso più oggettivo in difesa dell'istituzionalizzazione del matrimonio. Questo avviene con tutta probabilità perché il Matheolus viene messo in relazione con un altro poema di Jean Le Fèvre, il Livre de Leësce, che elogia e difende le virtù femminili. I due testi circolano assieme in quattro mss. che si inseriscono nel dibattito letterario e giuridico sul matrimonio.

17 Christine Reno et Inès villelA-PETIT, Du "Jeu des échecs moralisés" à Christine de Pizan: un recueil bien mystérieux (BnF, fr. 580), pp.263-276, attraverso un attento esame codicologico, rilevano l'omogeneità intellettuale del manoscritto Paris, BnF, fr. 580, nonostante la presenza, tra quattro testi di stampo morale e didattico, dell'Epistre à la Reine, opera a carattere politico di Christine de Pizan. Vengono riscontrati inoltre preziosi indizi sulla provenienza della raccolta e sul committente, che potrebbe ragionevolmente essere Jean de Berry.

18 Anne schoysman, Recueil d'auteur, recueil thématique? Le cas de la diffusion manuscrite de textes sur le thème de la «vraie noblesse» traduits par Jean Miélot, pp. 277-287, studiando la diffusione dei manoscritti contenenti il Debat d'Honneur (traduzione di Miélot dell'Opusculum de presidencia dell'umanista italiano Giovanni Aurispa) e il Debat de vraie noblesse (traduzione del De nobilitate di Buonaccorso da Pistoia), osserva come il nome di Miélot appaia solo nei recueils d'auteur (Bruxelles, KBR 9778-80; Copenhague, Thott 1090), dove, in assenza di un'unità tematica, la coerenza è data proprio dalla presenza anche iconografica - del translateur. Nelle raccolte costituite secondo criteri tematici (KBR, 104-93-97; KBR 10977-79; BnF fr. 1968; BnF fr. 5413), invece, manca qualunque riferimento a Miélot.

René sTUIP, Unité de l'enluminure, unité du manuscrit? À propos d'un manuscrit contenant une copie des "Heures de Contemplacion" de Christine de Pizan (La Haye, KB, 73 J 55), pp. 289-301, si interessa alle due copie conosciute dell'Histoire de Contemplacion, inserite in raccolte organiche di opere prevalentemente didattiche. In particolare il ms. La Haye, KB, 73 J 55 ha un tono morale e religioso che ne dimostrerebbe l'unitarietà. Ma un'attenta disamina codicologica rivela che si possono distinguere nel volume tre unità materiali e due scritture diverse, mentre tutte le miniature sarebbero attribuibili ad uno stesso artista. Questo pone parecchie domande, prima fra tutte: le tre parti sono copiate indipendentemente e riunite in seguito casualmente o sono state commissionate a vari copisti secondo un progetto prestabilito? Quanto si conosce della storia del manoscritto non offre utili elementi di risposta. 
20 Martine THIRY-STASSIN, Un légendier propre pour les Blanches Dames de Namur (XVe siècle), pp.303-313, esamina le condizioni di produzione, la materialità e la struttura del contenuto del manoscritto Bruxelles, KBR II 2243. Esso contiene vite e leggende dei santi della famiglia religiosa dei Carmelitani alla quale appartenevano le Blanches Dames di Namur cui era destinato, e soddisfa quindi pienamente le attese delle religiose.

21 Lori J. WALteRs, Le thème du livre comme don de sagesse dans le ms. Paris, BnF, fr. 926, pp. 315-331, prende in considerazione il manoscritto prodotto per Marie, figlia del duca Jean de Berry, dal suo confessore Jean de Courcy. Le quattro opere morali e politiche contenute hanno lo scopo di formare Marie e sono una summa di saggezza. La raccolta è ispirata dalle concezioni di Gerson e Christine de Pizan, che contribuiscono a formare l'immagine della nazione Francia, di cui le donne sono la migliore incarnazione.

22 Frank WillaERT, Les Opera Omnia d'une mystique brabançonne. Réflexions sur la mise en recueil et la tradition manuscrite des cuvres de Hadewijck (d'Anvers?), pp. 333-345, analizza i quattro manoscritti che contengono la totalità delle opere della mistica originaria del Brabante (14 visioni, 31 lettere, 46 canzoni e 16 lettere rimate). Difficile stabilire se queste collezioni siano state riunite dalla stessa Hadewijck; è possibile invece affermare che la mano organizzatrice della beghina è responsabile della strutturazione di ognuna delle collezioni. 